\title{
Moderação inteligente de mensagens em ambientes virtuais de aprendizagem para alunos privados de liberdade
}

\author{
Antonio Leandro Martins Candido ${ }^{1}$, Corneli Gomes Furtado Júnior ${ }^{1}$, \\ Francisco Aislan da Silva Freitas ${ }^{1}$ \\ ${ }^{1}$ Programa de Pós-Graduação em Ciência da Computação - PPGCC \\ Instituto Federal de Educação, Ciência e Tecnologia do Ceará - IFCE \\ Av. Treze de Maio, 2081 - Benfica - 60.040-215 - Fortaleza - CE - Brasil \\ \{antoniolmcandido, junior.corneli83, aislansf\}@gmail.com
}

\begin{abstract}
Virtual learning environments used by incarcerated student must prevent inappropriate communication between those involved in the teaching and learning process. Due to the potentially large number of messages, intelligent moderation of such communication is necessary. In this article, a model for intelligent moderation of AVA messages for incarcerated student is proposed and analyzed. The results indicate that the best performance in message classification reaches values of up to $94.28 \%$ for the metric $F$ measure.
\end{abstract}

Resumo. Ambientes virtuais de aprendizagem utilizados por alunos privados de liberdade devem impedir a comunicação indevida entre os envolvidos no processo de ensino e aprendizagem. Devido ao número potencialmente elevado de mensagens, faz-se necessário a moderação inteligente dessa comunicação. Nesse artigo é proposto e analisado um modelo para moderação inteligente de mensagens em AVA para alunos com restrição de liberdade. Os resultados indicam que o melhor desempenho na classificação de mensagens alcança valores de até 94,28\% para a métrica F-measure.

\section{Introdução}

O sistema penitenciário brasileiro é assunto de preocupação nacional, principalmente ao levar em conta que, com uma população de 200 milhões de habitantes, o Brasil tem mais de 700 mil pessoas vivendo em prisões [Brasil 2016]. A situação da maioria dos presídios é de superlotação, de pouca verba e infraestrutura insuficiente.

Intuitivamente é dedutível que a população prisional no Brasil tenha menor grau de escolaridade e os números confirmam essa impressão. Segundo o relatório do Ministério da Justiça [Brasil 2016], manter os jovens na escola pelo menos até o término do ensino fundamental pode ser uma das políticas de prevenção mais eficientes para a redução da criminalidade e, por conseguinte, da população prisional.

O Ensino a Distância (EaD), modalidade na qual alunos e professores estão separados, física ou temporalmente surgiu em decorrência da necessidade de proporcionar educação aos segmentos da população não adequadamente servidos pelo sistema tradicional de ensino. Esta modalidade de ensino, mediada por tecnologias digitais, pode ser utilizadas para a formação da população prisional como medida de ressocialização [Ferreira 2016]. 
Entretanto, os diversos Ambientes Virtuais de Aprendizagem (AVA) disponíveis [Moodle 2019] [TelEduc 2019] [Amadeus 2019] [Dokeos 2019] não ofertam ferramentas que permitam a moderação inteligente das mensagens, existentes em chats e fóruns, por exemplo, realizadas entre alunos, tutores e professores. Assim, o uso do AVA pode ser desvirtuado para realização de comunicação indevida (comunicação externa ao presídio ou entre presos, por exemplo), facilitando a prática de delitos, mesmo que o aluno esteja sob a tutela do Estado.

Nesse trabalho é proposto um modelo inteligente para a moderação das trocas de mensagens entre os atores que compõem o ensino a distância para pessoas restritas de liberdade e analisado o desempenho dos classificadores ${ }^{1}$ Naive Bayes [Mooney et al. 1998] e SVM (do inglês, Support Vector Machine - SVM) [Ma e Guo 2014], combinados com as técnicas de pré-processamento remoção de stopwords ${ }^{2}$ e stemming e as técnicas de extração de características Count Vectorizer - CV e TF-IDF (do inglês Term Frequency - Inverse Document Frequency) [Salton e Buckley 1988], utilizados na proposta.

No sistema de moderação inteligente, toda a comunicação relacionada à aula ou à disciplina deve ocorrer normalmente, enquanto as não relacionadas ou consideradas inadequadas devem ser impedidas de chegar ao destinatário. Ao tutor deve ser possível o tratamento de falsos-positivos ${ }^{3}$.

Incorporado ao AVA, o sistema de moderação inteligente pode contribuir com a formação e desenvolvimento de competências, com a ressocialização de alunos com restrição de liberdade e, de maneira indireta, com a redução do número de reincidências em crimes.

Os testes apresentados nesse trabalho foram simulados com dados reais de uma turma do curso Técnico em Informática na modalidade a distância do Instituto Federal de Educação, Ciência e Tecnologia do Ceará - IFCE. Os resultados obtidos permitem a classificação de mensagens com valores de até 94,28\% para a métrica F-measure.

Esse trabalho está organizado da seguinte maneira: após a introdução realizada na Seção I, os trabalhos relacionados são apresentados na Seção II. O modelo para a moderação inteligente e a metodologia de validação são apresentados na seção III. Os resultados são apresentados na seção IV. Por fim, as considerações finais são apresentadas na seção V.

\section{Trabalhos Relacionados}

Diversos são os trabalhos relacionados ao problema da classificação de textos educacionais, porém até o presente momento não foram encontrados trabalhos que abordassem a classificação para moderação inteligente de mensagens em AVA para alunos privados de liberdade.

Com a utilização do classificador Naive Bayes e Análise Semântica Latente (do inglês Latent Semantic Analysis - LSA) em um fórum de um AVA, Lui et al. (2007)

\footnotetext{
1 Classificadores são dispositivos inteligentes utilizados para inferir propriedades e atributos sobre informações até então desconhecidas [Mitchell 1997].

${ }^{2}$ Stopwords são palavras que não influenciam diretamente no processo de classificação, como por exempo, pronomes e artigos.

${ }^{3}$ Falsos-positivos são mensagens adequadas classificadas como inadequadas.
} 
VIII Congresso Brasileiro de Informática na Educação (CBIE 2019)

Anais do XXX Simpósio Brasileiro de Informática na Educação (SBIE 2019)

avaliam o nível de participação dos alunos, atribuem uma nota às mensagens consideradas relevantes e identificam de quais categorias as mensagens pertencem, porém realizam seus experimentos em apenas um cenário de testes.

Ravi e Kim (2007) utilizam SVM para classificar as mensagens dos alunos em dois grupos diferentes, o grupo das perguntas e o grupo das respostas. O classificador alcança precisões de $88 \%$ para as categorias de perguntas e $73 \%$ para a categoria de respostas. É possível alcançar valores mais altos de precisão se utilizar TF-IDF no processo de extração de características.

Scheuer e McLaren (2008) afirmam que o SVM, Árvores de Decisão Aprimorada e Listas de Decisão são os mais efetivos dentre os seis algoritmos de aprendizado de máquina que utilizam para a classificação das mensagens de fóruns em duas categorias, Boas Discussões ou Más Discussões. Comparam o desempenho dos classificadores com os julgamentos dos professores e conseguem valores Kappa [Landis e Koch 1977] variando entre $60 \%$ e $71 \%$.

Lin et al. (2009) propõem um Sistema Classificador de Gêneros utilizando Árvores de Decisão, cujo propósito é identificar a quais dos gêneros as mensagens enviadas pelos alunos pertencem. As mensagens podem ser classificadas como anúncios, perguntas, interpretações, conflitos, afirmações ou outros. Um aspecto interessante é que a ferramenta é integrada em um AVA, porém a classificação não acontece em tempo real.

Azevedo et al. (2011) realizam uma análise qualitativa das contribuições textuais registradas por alunos em fóruns de discussão. Utilizam o software MineraFórum aplicado em dez diferentes fóruns e realizam as comparações das análises feitas pelo software e pelos professores. Obtêm bons valores de precisão alcançando até 96,43\%.

O trabalho de Oliveira Junior e Esmin (2011) é desenvolver uma ferramenta para classificar as mensagens entre positivas ou negativas. Fazem uma comparação entre os algoritmos SVM e Naive Bayes, o SVM se destaca com precisão de $71 \%$ em relação aos $64 \%$ do Naive Bayes. Propõem uma ferramenta de monitoramento automático de mensagens, entretanto a classificação não acontece em tempo real, é acionada uma vez por dia através de um agendamento no sistema operacional.

Rolim et al. (2016) propõem uma solução computacional para classificar as mensagens de um fórum em três categorias, dúvidas, postagens neutras ou respostas. Utilizam três técnicas de classificação, Naive Bayes, Árvores de Decisão e a rede neural Perceptron Multicamadas (do inglês Multilayer Perceptron - MLP) [Kruse et al. 2013] alcançam valores altos de precisão chegando até 97\% com a utilização da MLP.

Ferreira et al. (2017) realizam um trabalho para identificar o nível de colaboração dos alunos nos fóruns, utilizam SVM alcançaram precisões de 80\%, 68\%, $60 \%$ e $84 \%$ para as categorias presença de grupo, elogio/apreciação, introdução de novas perspectivas e partilhamento de informações, respectivamente.

Existem técnicas comuns utilizadas no pré-processamento nestes trabalhos, a remoção das stopwords e a realização do stemming, que reduz as palavras ao seu tronco, base ou raiz. E alguns utilizaram algoritmos para a segmentação dos termos em ngramas, contadores de frequência de termos, como o CV e o parâmetro indicador TFIDF. Na Tabela 1 é possível ver quais técnicas foram utilizadas nos trabalhos relacionados. 
VIII Congresso Brasileiro de Informática na Educação (CBIE 2019)

Anais do XXX Simpósio Brasileiro de Informática na Educação (SBIE 2019)

Tabela 1. Técnicas utilizadas nos trabalhos relacionados

\begin{tabular}{|c|c|c|}
\hline Autores & $\begin{array}{l}\text { Algoritmo de } \\
\text { Classificação }\end{array}$ & $\begin{array}{c}\text { Pré-processamento e } \\
\text { Extrações de características }\end{array}$ \\
\hline Lui et al., 2007 & LSA e Naive Bayes & Stopwords e Stemming \\
\hline Ravi e Kim, 2007 & SVM & $\begin{array}{c}\text { Stopwords, Stemming e } \\
\text { N-gramas }\end{array}$ \\
\hline $\begin{array}{l}\text { Scheuer e McLaren, } \\
2008\end{array}$ & $\begin{array}{c}\text { Árvores de Decisão, Listas de } \\
\text { Decisão e SVM }\end{array}$ & $\begin{array}{c}\text { Stopwords, Stemming e } \\
\text { N-gramas }\end{array}$ \\
\hline Lin et al., 2009 & Árvores de Decisão & Stopwords, N-gramas e TF-IDF \\
\hline Azevedo et al., 2011. & Grafos & Stopwords e Stemming \\
\hline Oliveira Junior e Esmin, & Naive Bayes e SVM & Stopwords \\
\hline Rolim et al., 2016 & $\begin{array}{c}\text { Árvores de Decisão, Naive } \\
\text { Bayes e MLP }\end{array}$ & $\begin{array}{c}\text { Stopwords, Stemming, CV e } \\
\text { TF-IDF }\end{array}$ \\
\hline Ferreira et al., 2017 & SVM & TF-ISF \\
\hline
\end{tabular}

Os classificadores SVM e Naive Bayes obtiveram bons desempenhos nas classificações de textos. Partindo desse ponto são feitas comparações entre o SVM e o Naive Bayes com e sem as técnicas para o pré-processamento e extração de características, com o intuito de identificar quais combinações possuem melhores desempenhos na classificação dos textos disponibilizados no ambiente virtual de aprendizagem escolhido neste trabalho.

\section{Modelo para a moderação inteligente e metodologia de validação}

Nesse trabalho, o modelo proposto para moderação de mensagens é composto por três etapas. Na etapa inicial, é necessário treinar o classificador como rotular as mensagens. Esse treinamento ocorre a partir de uma base de mensagens preexistentes, rotuladas enquanto pertinentes ou não pertinentes ao assunto abordado em uma disciplina.

Após a etapa de treinamento, há a etapa de testes, nesse processo são avaliados os desempenhos dos classificadores. $\mathrm{Na}$ última etapa as novas mensagens não rotuladas enviadas pelos alunos são rotuladas de acordo com a predição indicada na fase anterior e são reutilizadas para incrementar a base de treinamentos, incrementando o número de mensagens conhecidas pelo classificador e, consequentemente, fortalecendo o processo de treinamento. Esse processo de incremento compõe o reaprendizado do classificador.

Por fim, as mensagens rotuladas enquanto pertinentes são entregues ao destinatário, enquanto as não pertinentes devem ser retidas até análise de um responsável (o tutor, por exemplo). Isso permite que mensagens classificadas erroneamente sejam entregues ao destinatário e contribuam para a melhora de desempenho no processo de classificação.

\subsection{Cenários de Testes}

Os dois classificadores SVM e Naive Bayes associados com as técnicas de extração de características são aplicados cada um em quatro diferentes cenários.

No cenário 1 nenhuma técnica de pré-processamento é aplicada na base de dados. São realizadas as classificações utilizando o CV com o Naive Bayes, depois TFIDF com Naive Bayes, CV com SVM e por fim, TF-IDF com SVM. 
VIII Congresso Brasileiro de Informática na Educação (CBIE 2019)

Anais do XXX Simpósio Brasileiro de Informática na Educação (SBIE 2019)

O cenário 2 conta apenas com a técnica de pré-processamento de remoção das stopwords aplicada na base de dados. Em sequência são realizadas as classificações utilizando as técnicas de extração de características associadas com os classificadores conforme no cenário 1 .

No cenário 3 apenas a técnica de pré-processamento de stemming é aplicada na base de dados e da mesma forma que nos cenários anteriores, são realizadas as classificações.

Por fim, no cenário 4 a base de dados é pré-processada com as duas técnicas, remoção das stopwords e aplicação de stemming. Logo após são realizadas as classificações. Na Tabela 2 é possível ver a distribuição dos cenários de testes.

Tabela 2. Distribuição dos Cenários de Testes

\begin{tabular}{llll} 
Cenários & \multicolumn{1}{c}{$\begin{array}{c}\text { Classificadores } \\
\text { com CV ou TF-IDF }\end{array}$} & & \multicolumn{2}{c}{$\begin{array}{c}\text { Técnicas de Pré- } \\
\text { processamento }\end{array}$} \\
Cenário 1 & $\begin{array}{l}\text { Naive Bayes } \\
\text { Stopwords }\end{array}$ & Stemming \\
& SVM & & \\
\hline \multirow{2}{*}{ Cenário 2 } & $\begin{array}{l}\text { Naive Bayes } \\
\text { SVM }\end{array}$ & $\mathrm{x}$ & \\
\hline \multirow{2}{*}{ Cenário 3 } & $\begin{array}{l}\text { Naive Bayes } \\
\text { SVM }\end{array}$ & & $\mathrm{x}$ \\
\hline \multirow{2}{*}{ Cenário 4 } & $\begin{array}{l}\text { Naive Bayes } \\
\text { SVM }\end{array}$ & $\mathrm{x}$ & $\mathrm{x}$ \\
\hline
\end{tabular}

\subsection{Construção da base de dados}

Para conseguir classificar automaticamente novas mensagens enviadas ao fórum é necessário construir uma base de dados padrão contendo as mensagens adequadas e as inadequadas às disciplinas, classificadas por um especialista.

Neste trabalho são agrupadas duas bases de dados para a construção da base de dados padrão, uma contendo 736 mensagens consideradas adequadas, coletadas do AVA da disciplina de Fundamentos de Informática do curso à distância de Técnico em Informática do IFCE e outra contendo 480 mensagens consideradas inadequadas, com palavras de vocabulário comum entre pessoas privadas de liberdade encontradas em notícias de sites policiais, jornais e documentários, totalizando 1216 mensagens distribuídas entre as duas classes e separadas aleatoriamente em dois conjuntos de treinamento e teste.

No processo de moderação de novas mensagens com a utilização do modelo de classificação proposto, são utilizadas 304 novas mensagens não rotuladas.

\subsection{Pré-Processamento}

No pré-processamento são aplicadas duas técnicas: remoção das stopwords e stemming. $\mathrm{Na}$ Figura 1 (a) percebe-se a predominância das palavras na base de dados sem a aplicação da técnica de remoção de stopwords, a predominância dos artigos e pronomes 
VIII Congresso Brasileiro de Informática na Educação (CBIE 2019)

Anais do XXX Simpósio Brasileiro de Informática na Educação (SBIE 2019)

interfere na classificação. Na Figura 1 (b) é possível ver a predominância das palavras quando utilizada a técnica de remoção de stopwords.

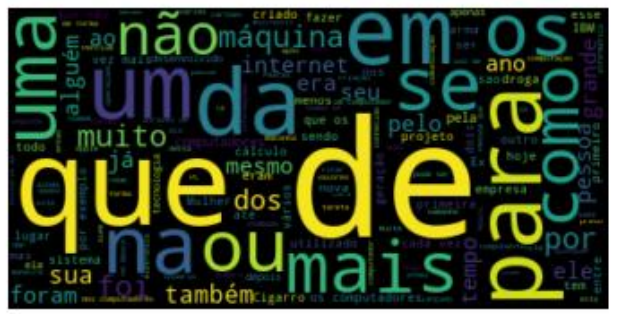

Figura 1 (a). Predominância das palavras com as stopwords

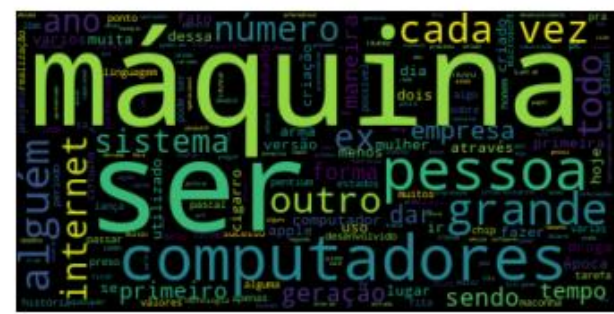

Figura 1 (b). Predominância das palavras sem as stopwords

Com a aplicação da técnica de stemming as palavras flexionadas ou derivadas são reduzidas à raiz, para facilitar na identificação de similaridade entre as palavras no processo de classificação. Para executar as técnicas de pré-processamento é utilizado o Kit de Ferramentas de Linguagem Natural (do inglês Natural Language Toolkit NLTK) desenvolvido em linguagem de programação Python e com documentação disponível [NLTK 2019].

\subsection{Extração de Características}

Os algoritmos de extração de características são utilizados para selecionar palavras que possuem mais representatividade na base de dados. Neste trabalho são utilizados dois deles isoladamente e em cenários diferentes, o contador de frequência de termos $\mathrm{CV}$ e o TF-IDF.

Com a utilização do $\mathrm{CV}$ a base de dados é convertida em uma matriz esparsa com a frequência de cada termo (palavra) representada por tokens, são utilizados parâmetros para definir um limite máximo e mínimo de frequência para os termos a serem selecionados. Um exemplo de matriz construída pelo CV pode ser vista na Figura 2 , onde cada termo recebe a sua quantidade de frequência em cada uma das mensagens.

\begin{tabular}{|lccccc|}
\hline & Termo1 & Termo2 & Termo3 $\ldots$ & TermoN \\
mensagem1 & 5 & 6 & 1 & 0 \\
mensagem2 & 0 & 0 & 0 & 1 \\
mensagemN & 1 & 2 & 2 & 0 \\
\hline
\end{tabular}

Figura 2. Exemplo de uma matriz construída com a aplicação do Count Vectorizer

O limite máximo de frequência dos termos nas mensagens é de até $70 \%$, para não serem selecionados termos comuns em ambas as classes e o limite mínimo é de pelo menos duas frequências, com o propósito de remover termos com pouca relevância para a classificação. Esses limites foram definidos após vários testes, quando alcançaram melhores resultados na classificação.

O TF-IDF é utilizado para calcular a frequência do termo do diálogo pelo inverso da frequência do termo em relação a todos os diálogos, ele é utilizado como uma medida estatística por meio da equação (1). 


$$
w_{i, j}=t f_{i, j} * \log \left(\frac{N}{d f_{i}}\right)
$$

Vale ressaltar que $t f_{i, j}$ é número de frequência do termo $i$ na mensagem $j, d f_{i}$ é o número de mensagens contendo o termo $i$ e $N$ o número total de mensagens. Os termos foram ranqueados e selecionados apenas os primeiros $20 \%$ para garantir um conjunto de termos com melhor relevância para a classificação.

\subsection{Classificação}

Primeiro é realizada a classificação da base de dados padrão, com uma divisão em $70 \%$ das mensagens para o treino, onde os parâmetros de cada classificador são ajustados e depois são realizados os testes com os $30 \%$ restantes das mensagens. Neste trabalho é aplicada a técnica de validação cruzada K-folds com uma divisão em cinco subconjuntos para ter uma melhor estimativa de classificação.

Em um segundo momento é a realizada a classificação das novas mensagens enviadas ao fórum e não rotuladas, em que cada mensagem recebe o rótulo de acordo com a predição do classificador e essa mensagem é inserida na base de treino para a reaprendizagem do classificador.

$\mathrm{Na}$ avaliação dos desempenhos dos classificadores são usadas as métricas de Precisão, Recall e F-measure [Hripcsak e Rothschild 2005]. A Precisão é utilizada para avaliar a quantidade de instâncias que são classificadas corretamente, enquanto que o Recall para avaliar a frequência em que o classificador encontra os exemplos de uma classe e a F-measure é utilizada como uma média harmônica da Precisão e do Recall. São realizadas 500 iterações de treinamentos, testes e validações e calculadas as médias e desvios padrões de cada métrica.

\section{Resultados}

Após a remoção das stopwords, um aspecto interessante ao usar o CV é que ele é capaz de remover outros termos que ainda possuíam bastante frequência, entretanto não representavam claramente uma classe, como por exemplo, a palavra "hoje", é um termo que não é removido no pré-processamento, porém é bastante comum encontrá-lo em diálogos tanto adequados, como inadequados.

Com a utilização do CV ao invés do TF-IDF, os classificadores recebem uma quantidade maior de dados para classificar (termos selecionados entre os diálogos), pois de acordo com um dos parâmetros, termos com pelo menos duas ocorrências nos diálogos são mantidos no conjunto de dados para a classificação. Por conta disso o classificador SVM tem uma pequena desvantagem em relação ao Naive Bayes, por não possuir melhor desempenho para conjunto de dados maiores.

Além de passar para os classificadores um conjunto de dados menor, o TF-IDF consegue extrair os termos com maior relevância na base de dados sem a necessidade das funções de pré-processamento, contudo ele também é aplicado em conjunto com as funções para averiguar qual alteração no desempenho dos classificadores ocorre com essa combinação.

$\mathrm{Na}$ Tabela 3 a seguir é possível ver as médias das métricas com seus respectivos desvios padrões dos dois classificadores nos quatro cenários de testes. A comparação dos desempenhos leva em consideração a métrica F-measure. Analisando os resultados 
VIII Congresso Brasileiro de Informática na Educação (CBIE 2019)

Anais do XXX Simpósio Brasileiro de Informática na Educação (SBIE 2019)

é possível observar que para o cenário 1, o classificador Naive Bayes obtém melhor desempenho em relação ao classificador SVM quando utilizado o CV para a extração de características.

Tabela 3. Resultados dos classificadores NB (Naive Bayes) e SVM nos Cenários de Testes: C1, C2, C3 e C4

\begin{tabular}{llcccccc} 
& & \multicolumn{2}{c}{ Precisão } & \multicolumn{2}{c}{ Recall } & \multicolumn{2}{c}{ F-measure } \\
\cline { 2 - 7 } & & CV & TF-IDF & CV & TF-IDF & CV & TF-IDF \\
\hline \multirow{2}{*}{ C1 } & NB & $94,32 \pm 1,46$ & $92,93 \pm 1,25$ & $92,34 \pm 1,64$ & $83,57 \pm 2,29$ & $93,21 \pm 1,49$ & $86,49 \pm 2,19$ \\
& SVM & $91,57 \pm 1,77$ & $94,46 \pm 1,28$ & $93,04 \pm 1,55$ & $90,52 \pm 1,93$ & $92,20 \pm 1,60$ & $92,12 \pm 1,65$ \\
\hline \multirow{2}{*}{ C2 } & NB & $94,32 \pm 1,48$ & $93,04 \pm 1,21$ & $92,33 \pm 1,68$ & $83,69 \pm 2,22$ & $93,31 \pm 1,52$ & $86,61 \pm 2,13$ \\
& SVM & $91,44 \pm 1,93$ & $94,45 \pm 1,29$ & $92,84 \pm 1,66$ & $90,45 \pm 1,97$ & $92,04 \pm 1,74$ & $92,08 \pm 1,68$ \\
\hline \multirow{2}{*}{ C3 } & NB & $94,66 \pm 1,40$ & $93,08 \pm 1,34$ & $92,61 \pm 1,64$ & $84,36 \pm 2,37$ & $93,52 \pm 1,48$ & $87,17 \pm 2,27$ \\
& SVM & $91,74 \pm 1,80$ & $94,81 \pm 1,33$ & $93,00 \pm 1,61$ & $92,06 \pm 2,54$ & $92,29 \pm 1,65$ & $93,19 \pm 1,99$ \\
\hline \multirow{2}{*}{ C4 } & NB & $94,64 \pm 1,37$ & $93,17 \pm 1,29$ & $92,59 \pm 1,62$ & $84,44 \pm 2,29$ & $93,50 \pm 1,45$ & $87,07 \pm 2,18$ \\
& SVM & $91,53 \pm 1,80$ & $95,31 \pm 1,40$ & $92,89 \pm 1,61$ & $93,88 \pm 2,01$ & $92,12 \pm 1,63$ & $94,28 \pm 1,88$ \\
\hline
\end{tabular}

Nos resultados dos testes do cenário 2 é possível perceber que o classificador Naive Bayes obtém uma melhora no desempenho ao utilizar a remoção das stopwords em conjunto com o CV, por outro lado, o desempenho do SVM para essa combinação é menor. O SVM continua apresentando melhor desempenho com a utilização do TF-IDF para esse segundo cenário com remoção das stopwords.

Percebe-se um destaque do classificador Naive Bayes ao utilizar apenas stemming no pré-processamento em conjunto com CV no cenário 3 e também um ganho de desempenho utilizando stemming e TF-IDF. Com a utilização do stemming as palavras foram reduzidas a raiz, o que ocasiona que alguns termos fiquem com a mesma estrutura, portanto há mais reduções no conjunto de dados.

No último cenário, com a utilização dos dois algoritmos de pré-processamento, remoção de stopwords e stemming, o classificador Naive Bayes e SVM mantém bons desempenhos com a utilização do $\mathrm{CV}$, entretanto, observa-se novamente que apenas o SVM consegue manter um bom desempenho utilizando o TF-IDF. Ao optar por utilizar o classificador Naive Bayes em um modelo de classificação, a melhor configuração do modelo é com stemming no pré-processamento e CV na extração de características.

Dependendo do tamanho da base de dados em que são aplicados esses dois classificadores, o uso do algoritmo de extração de características TF-IDF pode ganhar destaque no critério tempo de execução e o classificador SVM sempre mantém um bom desempenho por conta do conjunto de dados ser menor para a classificação.

\section{Considerações Finais}

Este trabalho apresenta um modelo de moderação inteligente capaz de classificar as mensagens enviadas em um fórum de um AVA como adequadas ou inadequadas. As mensagens inadequadas são consideradas aquelas contendo comunicação inapropriada à disciplina, frases contendo palavras utilizadas em gírias, ameaças, obscenidade, 
VIII Congresso Brasileiro de Informática na Educação (CBIE 2019)

Anais do XXX Simpósio Brasileiro de Informática na Educação (SBIE 2019)

xingamentos e alguns termos com significados implícitos, comuns do vocabulário utilizado por pessoas privadas de liberdade.

Ao analisar os desempenhos dos dois classificadores é possível concluir que o Naive Bayes tem desempenho superior apenas com a utilização do algoritmo Counter Vectorize na extração das características da base de dados, com o uso do algoritmo TFIDF, destaca-se o classificador SVM. Esse é um ponto importante observado, pois o modelo adotado deve ser capaz de classificar os diálogos em um breve período de tempo, para que não interfira no fluxo do uso do fórum em um AVA e o algoritmo de extração de características TF-IDF contribui tornando o processo de classificação mais rápido em relação ao $\mathrm{CV}$.

Como trabalho futuro pretende-se incorporar o modelo inteligente de moderação em um ambiente real utilizando o classificador SVM com remoção de stopwords, stemming e TF-IDF em um fórum do Moodle, com a função principal de classificar automaticamente cada mensagem enviada ao fórum e evitar o envio das mensagens inadequadas, exibindo alertas visíveis somente aos tutores com informações do usuário e da mensagem que foi impedida de ser enviada, permitindo optar por enviar a mensagem ou não, pretende-se também estender os testes e registrar os resultados em novos artigos.

\section{Referências}

Amadeus (2019). Amadeus documentation - disponível em https://softwarepublico.gov.br/social/amadeus. acesso em: 03 de março de 2019.

Azevedo, B. F. T., Behar, P. A., Reategui, E. B. (2011). Análise das mensagens de fóruns de discussão através de um software para mineração de textos. In: Simpósio Brasileiro de Informática na Educação, p. 20-29.

Brasil, Ministério da Justiça (2016). Departamento Penitenciário Nacional: Infopen Levantamento Nacional de Informações Penitenciárias. Disponível em: http://dados.gov.br/dataset/infopen-levantamento-nacional-de-informacoespenitenciarias1. acesso em: 14 de abril de 2019.

Dokeos (2019). Dokeos documentation - disponível em https://www.dokeos.com/. acesso em: 03 de março de 2019.

Ferreira, M. A. D., Ferreira, R., Cavalcanti, A. P., Carvalho, R. e Sebastião Neto (2017). Mineração de Texto Aplicada à Identificação de Colaboração em Fóruns Educacionais. In: Simpósio Brasileiro de Informática na Educação, p. 1437-1446.

Ferreira, M. M. (2016). Educação à distância para sistemas prisionais: um estudo sobre viabilidades técnicas de infraestrutura necessária para implementação da educação em rede nas escolas do sistema prisional. Universidade Federal de Minas Gerais: Dissertação (Mestrado) - Faculdade de Educação.

Hripcsak, George e Rothschild, Adam S. (2005). Agreement, the F-Measure, and Reliability. In Information Retrieval Journal of the American Medical Informatics Association. 12(3).

Kruse, R., Borgelt, C., Klawonn, F., Moewes, C., Steinbrecher, M., e Held, P. (2013). Multilayer perceptrons. In Computational Intelligence, p. 47-81. Springer. 
VIII Congresso Brasileiro de Informática na Educação (CBIE 2019)

Anais do XXX Simpósio Brasileiro de Informática na Educação (SBIE 2019)

Landauer, T.K. et al., (1998). An Introduction to Latent Semantic Analysis", Discourse Processes, 25(2-3), 337-354.

Larocca N. J., Santos, A. D., Kaestner, A. A. C, e Freitas, A. A. (2000). Generating Text Summaries through the Relative Importance of Topics. In: the Proceedings of the International Joint Conference IBERAMIA/SBIA, Atibaia, SP.

Landis, J.R., Koch, G.G. (1977). The Measurement of Observer Agreement for Categorical Data. Biometrics 33, 159-174.

Lin, F., Hsieh, L., Chuang, F. (2009). Discovering Genres of Online Discussion Threads via Text Mining. In: Computers \& Education, p. 481-495.

Lui, A. K., Li, S. C., Choy, S. O. (2007). An Evaluation of Automatic Text Categorization in Online Discussion Analysis. In: IEEE International Conference on Advanced Learning Technologies.

Ma, Yunqian e Guo, Guodong (2014). Support Vector Machines Applications. Springer International Publishing, New York.

Mitchell, T. M. (1997). Machine Learning. 1.ed. New York: McGraw-Hill, Inc.

Moodle (2019). Moodle documentation - disponível em http://www.moodle.org. acesso em: 07 de abril de 2019.

Mooney, R. J., Bennett, P. N., e Roy, L. (1998). Book recommending using text categorization with extracted information. In Papers of the AAAI-98/ICML-98 Workshop on Learning for Text Categorization and Papers of the AAAI-98 Workshop on Recommender Systems., Madison,WJ.

NLTK. (2009). Natural Language Toolkit - disponível em https://www.nltk.org/ acesso em: 02 de abril de 2019.

Oliveira Júnior, R. L. de, Esmin, A. A. A. (2012). Monitoramento Automático de Mensagens de Fóruns de Discussão Usando Técnica de Classificação de Texto SemiSupervisionado. In: Simpósio Brasileiro de Informática na Educação.

Ravi, S., Kim, J. (2007). Profiling Student Interactions in Threaded Discussions with Speech Act Classifiers. In: Artificial Intelligence in Education.

Rolim, V. B., Ferreira, R., Costa, E. (2016). Identificação Automática de Dúvidas em Fóruns Educacionais. In: Simpósio Brasileiro de Informática na Educação. p. 936945.

Salton, G. e Buckley, C. Term-weighting approaches. In automatic text retrieval. Journal information processing and management: an international Journal, 24(5), 513-523.

Scheuer, O. e McLaren, B. M. (2008). Helping Teachers Handle the Flood of Data in Online Student Discussions. In: Intelligent Tutoring Systems, p. 323-332.

TelEduc (2019). TelEduc documentation - disponível em http://www.teleduc.org.br/. acesso em: 01 de abril de 2019. 